\title{
Equalização Cega de Canais Espaço-Temporais Variantes no Tempo usando Predição Linear Adaptativa em Sistemas OFDM
}

\author{
Alam Silva Menezes ${ }^{\dagger}$, Cristiano Magalhães Panazio ${ }^{\ddagger}$ e João Marcos Travassos Romano ${ }^{\dagger}$
}

\begin{abstract}
Resumo-Esse trabalho propõe o uso de predição linear progressiva para equalização de canais espaço-temporais variantes no tempo em sistemas OFDM. Por meio de simulações, indicamos que a predição linear obtém melhor desempenho que o algoritmo MERRY tanto para explorar a diversidade espacial quanto para rastrear as variações do canal ao longo do tempo.
\end{abstract}

Palavras-Chave-OFDM, Equalização Cega, Predição Multicanal, SIMO, MERRY, Estatísticas de Ordem 2, Encurtamento de Canal, Canais Variantes no Tempo.

Abstract-This paper introduce the forward linear prediction for equalization of OFDM systems in space time channels. We shown by simulations that linear prediction structure have better performance that MERRY algorithm both to exploit space time diversity than tracking channel variations along of time.

Keywords-OFDM, Blind Channel Shortening, Linear Multichannel Prediction, SIMO, MERRY, Second Order Statistics, Time-Varying Channel.

\section{INTRODUÇÃO}

A robustez a canais seletivos em freqüência, eficiência na utilização da capacidade do canal, bem como a facilidade de implementação em dispositivos de processamento digital de sinais são alguns dos fatores que têm levado a modulação em múltiplas portadoras (MCM em Inglês) a fazer parte da maioria dos padrões que regem as comunicações em banda larga da atualidade. Para citar alguns exemplos, temos os padrões WLAN IEEE 802.11x, Wireless Metropolitan Area Networks (WMAN) IEEE 802.16x, Digital Video Broadcasting (DVB), Digital Audio Broadcasting (DAB), Asymmetric Digital Subscribe Line (ADSL) e Power Line Communications (PLC).

O canal torna-se seletivo em freqüência quando a largura de banda empregada é maior que a banda de coerência do canal [1]. Nestes casos, os sistemas que utilizam modulação em uma única portadora são afetados com a interferência intersimbólica (IIS), gerada pela característica de dispersão temporal dos canais de comunicação seletivos em freqüência. As estruturas empregadas para mitigar a IIS em tais circunstâncias são os equalizadores. Por outro lado, a IIS pode ser eliminada nos sistemas MCM, utilizando-se um intervalo de guarda, denominado prefixo cíclico (CP), com comprimento maior que o espalhamento temporal máximo do canal. Desta forma, os

${ }^{\dagger}$ Laboratório de Processamento de Sinais para Comunicações, FEEC, UNICAMP, Campinas, Brasil. ${ }^{\ddagger}$ Laboratório de Comunicações e Sinais, Faculdade de Engenharia Elétrica, Escola Politécnica da Universidade de São Paulo. E-mails: alam@decom.fee.unicamp.br, cpanazio@lcs.poli.usp.br, romano@decom.fee.unicamp.br. Este trabalho foi financiado pela CAPES equalizadores temporais utilizados em sistemas MCM procuram tornar a dispersão da resposta do canal equivalente menor que o intervalo de guarda. Tais estruturas de equalização são encontradas na literatura com a denominação de channel shortening [2].

Para canais variantes no tempo, necessita-se de estruturas adaptativas para realizar o encurtamento do canal. A adaptação de tais estruturas pode ser feita de forma supervisionada, quando há uma sequiência de treinamento disponível, ou de forma cega, explorando algumas características do sinal conhecidas a priori na recepção.

Os critérios de equalização cegos mais difundidos na literatura são o de decisão direta (DD) e o do módulo constante (CM) [3]. No entanto, tais critérios não são apropriados para aplicação no contexto de modulação em múltiplas portadoras. Isto se deve a falta de um alfabeto finito do sinal no domínio do tempo e pelo fato da estatística do sinal se aproximar de uma Gaussiana. Para lidar com estes problemas, novos critérios cegos de equalização para sistema MCM foram propostos. Entre eles, vale citar o algoritmo MERRY [2] pelos bons resultados obtidos. Embora o algoritmo MERRY apresente convergência global no contexto single input single output (SISO) e seja de simples implementação, a atualização dos coeficientes de seu equalizador é realizada uma vez a cada símbolo OFDM, o que pode degradar seu desempenho em canais variantes no tempo. Ainda, no contexto single input multiple output (SIMO), dependendo da inicialização, o algoritmo MERRY é incapaz de explorar sempre a diversidade presente neste canal.

Neste artigo, propomos o uso da predição linear multicanal e adaptativa como estrutura de encurtamento de canais SIMO variantes no tempo. Verificamos por meio de simulações que, quando comparada ao algoritmo MERRY, nossa estrutura proporciona mais rápida convergência, explora de forma mais efetiva a diversidade espaço-temporal do canal SIMO, bem como apresenta desempenho superior quando aplicada em canais variantes no tempo.

Este artigo está estruturado da seguinte forma. Na seção II, introduzimos o modelo do sistema. Na seção III, apresentamos nossa proposta baseada em predição linear, progressiva e multicanal. A seção IV descreve resumidamente o algoritmo MERRY. A seção V descreve o modelo utilizado para gerar o desvanecimento rápido dos canais. Na seção VI são apresentados e discutidos os resultados. Por fim, a seção VII conclui o artigo e traça as perspectivas de futuros trabalhos. 


\section{Modelo Do Sistema}

As técnicas para equalização em sistemas OFDM avaliadas no presente artigo são projetadas para operarem em canais SIMO. Tais canais podem ser obtidos por meio de superamostragem ou pela utilização de múltiplas antenas na recepção. A Figura 1 apresenta o modelo derivado da utilização de uma antena para transmissão e duas antenas na recepção. Todavia, a derivação dos algoritmos se dá de forma generalizada, com uma única antena na transmissão e $P$ antenas na recepção.

Para transformar a convolução linear entre os símbolos OFDM e o canal em convolução circular, uma redundância de tamanho $\nu$ apropriado, denominada prefixo cíclico (CP), é inserida no início de cada símbolo OFDM. Desta maneira, é possível equalizar no domínio da freqüência apenas com um banco de ganhos complexos dados pela FFT do canal equivalente. No entanto, para se ter a convolução circular é necessário que $\nu$ seja maior que o comprimento da resposta ao impulso do canal. Se tal condição não for atendida, equalizadores no domínio do tempo $\mathbf{w}_{i}$ são empregados, fazendo com que a resposta do sistema equivalente da convolução do canal com o equalizador atenda a condição estabelecida.

Após a inserção do CP à seqüência $x_{i}^{k}$ e sua conversão para serial, tem-se:

$$
x(M k+i)=x(M k+i+N) \quad i \in\{0,1, \ldots, \nu-1\},
$$

em que $M=N+\nu$.

Os dados recebidos pela $p$-ésima antena podem ser modelados por:

$$
u_{p}(n)=\mathbf{h}_{p}^{T} \mathbf{x}(n)+r_{p}(n)
$$

onde $\quad \mathbf{h}_{p}=\left[h_{p}(0) h_{p}(1) \ldots h_{p}\left(L_{h}-1\right)\right]^{T}$ é o vetor de coeficientes do $p$-ésimo subcanal, $\mathbf{x}(n)=$ $\left[x(n) x(n-1) \ldots x\left(n-L_{h}+1\right)\right]^{T}$ é o vetor de dados transmitidos, $r_{p}(n)$ é o ruído aditivo Gaussiano branco, $L_{h}$ é o comprimento do $p$-ésimo subcanal mais longo e $n=M k+i$

A saída do $p$-ésimo subequalizador é dada por:

$$
y_{p}(n)=\mathbf{w}_{p}^{T} \mathbf{u}_{p},
$$

onde $\quad \mathbf{w}_{p}=\left[w_{p}(0) w_{p}(1) \ldots w_{p}\left(L_{w}-1\right)\right]^{T}$ é $\quad$ o $\quad p$ ésimo subequalizador de comprimento $L_{w}$ e $\mathbf{u}_{p}(n)=$ $\left[u_{p}(n) u_{p}(n-1) \ldots u_{p}\left(n-L_{w}+1\right)\right]^{T}$ é o vetor de entrada do $p$-ésimo subequalizador.

A seqüência de dados equalizados na entrada do bloco FFT é dada por:

$$
y(n)=\sum_{p=0}^{P-1} y_{p}(n)=\sum_{p=0}^{P-1} \mathbf{w}_{p}^{T} \mathbf{u}_{p}(n)=\mathbf{w}^{T} \mathbf{u}_{s}(n),
$$

em que

$$
\begin{gathered}
\mathbf{w}=\left[\mathbf{w}_{0}^{T}, \mathbf{w}_{1}^{T}, \ldots, \mathbf{w}_{P-1}^{T}\right]^{T}, \\
\mathbf{u}_{s}(n)=\left[\mathbf{u}_{0}^{T}(n), \mathbf{u}_{1}^{T}(n), \ldots, \mathbf{u}_{P-1}^{T}(n)\right]^{T} .
\end{gathered}
$$

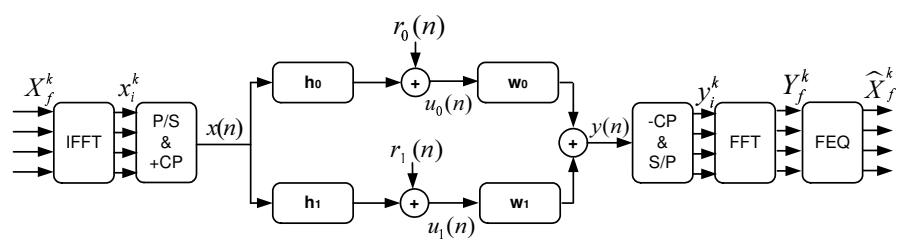

Fig. 1. Modelo do sistema. (I)FFT: transformada (inversa) rápida de Fourrier, $\mathrm{P} / \mathrm{S}$ : paralelo para serial, S/P: serial para paralelo, $+\mathrm{CP}$ : adiciona o prefixo cíclico, -CP: remove o prefixo cíclico, FEQ: equalizador no domínio da freqüência.

\section{PRedição Linear Muticanal}

As estruturas existentes para equalização autodidata baseada em estatísticas de ordem dois (EO2) podem ser dividas nas abordagens que utilizam a decomposição em subespaço e nas abordagens que empregam predição linear. A abordagem baseada em predição linear adaptativa multicanal [4] foi utilizada no presente artigo como estrutura destinada à equalização MCM no contexto SIMO.

A idéia é obter uma estimativa do vetor $\mathbf{u}(n)$, dado por:

$$
\mathbf{u}(n)=\left[\begin{array}{llll}
u_{0}(n) & u_{1}(n) & \ldots & u_{P-1}(n)
\end{array}\right]^{T},
$$

pela combinação das amostras passadas concatenadas no vetor $\mathbf{U}(n-1)=\left[\mathbf{u}^{T}(n-1) \mathbf{u}^{T}(n-2) \ldots \mathbf{u}^{T}\left(n-L_{w}+1\right)\right]^{T}$. As amostras do vetor predição podem ser escritas como:

$\widehat{\mathbf{u}}(n)=\mathbf{A}_{0}^{H} \mathbf{u}(n-1)+\mathbf{A}_{1}^{H} \mathbf{u}(n-1)+\ldots+\mathbf{A}_{L_{w}-1}^{H} \mathbf{u}\left(n-L_{w}+1\right)$

em que $\mathbf{A}_{i}$ é uma matriz de ordem $P \times P$. A equação (8) pode ser reescrita de uma forma compacta, dada por:

$$
\widehat{\mathbf{u}}(n)=\mathbf{F}^{H} \mathbf{U}(n-1) .
$$

A matriz $\mathbf{F}$ de ordem $P\left(L_{w}-1\right) \times L_{w}$ é dada por $\mathbf{F}=$ $\left[\begin{array}{llll}\mathbf{A}_{1} & \mathbf{A}_{2} & \ldots & \mathbf{A}_{L_{w}-1}\end{array}\right]^{T}$. Portanto, o vetor erro de predição é dado por:

$$
\begin{aligned}
\mathbf{e}(n) & =\mathbf{u}(n)-\widehat{\mathbf{u}}(n) \mid \mathbf{U}_{(n-1)} \\
& =\left[\begin{array}{ll}
\mathbf{I}_{A \times A} & -\mathbf{F}^{H}
\end{array}\right] \mathbf{U}(n),
\end{aligned}
$$

em que $\left.\widehat{\mathbf{u}}(n)\right|_{\mathbf{U}_{(n-1)}}$ é a estimativa do vetor $\mathbf{u}(n)$, dada as amostras do vetor $\mathbf{U}(n-1)$.

A matrix denominada matriz variância do vetor erro de predição de ordem $(P \times P)$ é definida por

$$
\mathbf{e}(n) \mathbf{e}^{H}(n)=\left[\mathbf{I}_{P \times P}-\mathbf{F}^{H}\right] \mathbf{R}_{\mathbf{U}}(n)\left[\mathbf{I}_{P \times P}-\mathbf{F}^{H}\right]^{H},
$$

em que $\mathbf{R}_{\mathbf{U}}(n)=\mathbf{U}(n) \mathbf{U}^{H}(n)$.

A minimização do traço da matriz variância do erro de predição conduz ao problema de otimização em função dos elementos da matriz $\mathbf{F}$, dado por:

$$
\min _{\mathbf{F}}\left[\mathbf{I}_{P \times P}-\mathbf{F}^{H}\right] \mathbf{R}_{\mathbf{U}}(n)\left[\mathbf{I}_{P \times P}-\mathbf{F}^{H}\right]^{H}=\sigma_{\mathbf{e}}^{2} \mathbf{I}_{P \times P} .
$$

O mesmo problema pode ser formulado pelas equações de Yule-Walker, chegando ao seguinte resultado:

$$
\left[I_{P \times P}-\mathbf{F}^{H}\right] \mathbf{R}_{\mathbf{U}}(n)=\left[\begin{array}{llll}
\mathbf{I}_{P \times P} \sigma_{\mathbf{e}}^{2} & \mathbf{0}_{P \times P} & \ldots & \mathbf{0}_{P \times P}
\end{array}\right] .
$$


A equação (13) pode ser reescrita com a matriz $\mathbf{R}_{\mathbf{U}}(n)$ particionada da seguinte forma:

$\left[\mathbf{I}_{P \times P}-\mathbf{F}^{H}\right]\left[\begin{array}{cc}\mathbf{r}_{0} & \mathbf{r} \\ \mathbf{r}^{H} & \mathbf{R}_{\mathbf{U}}(n-1)\end{array}\right]=\left[\begin{array}{lll}\mathbf{I}_{P \times P} \sigma_{\mathbf{e}}^{2} & 0_{P \times P} \ldots 0_{P \times P}\end{array}\right]$,

de onde se obtém as seguintes relações:

$$
\left\{\begin{array}{c}
\mathbf{F}=\mathbf{r R}_{\mathbf{U}}^{\dagger}(n-1) \\
\mathbf{I}_{P \times P} \sigma_{\mathbf{e}}^{2}=\mathbf{r}_{0}-\mathbf{r R}_{\mathbf{U}}^{\dagger}(n-1) \mathbf{r}^{H}
\end{array}\right.
$$

em que $(\cdot)^{\dagger}$ denota pseudoinversa. Verifica-se que tanto os elementos da matriz $\mathbf{F}$ quanto a matriz variância do erro de predição podem ser obtidos por EO2 do sinal cicloestacionário na entrada dos subequalizadores.

Conforme apresentado em [4], o problema de otimização (13) pode ser reescrito em função dos coeficientes do canal:

$$
\left[\mathbf{I}_{A \times A}-\mathbf{F}^{H}\right] \widehat{\mathbf{H}}=\mathbf{h}(0)\left[\mathbf{I}_{A \times A}-\mathbf{0}_{A \times L_{h}+L_{w}-1}\right],
$$

em que a matriz convolução do canal $\widehat{\mathbf{H}}$ de ordem $\left(P L_{w} \mathbf{x}\right.$ $\left.L_{h}+L_{w}-1\right)$ é dada por:

$$
\widehat{\mathbf{H}}=\left[\begin{array}{ccccc}
\mathbf{h}(0) & \cdots & \mathbf{h}\left(L_{h}-1\right) & & 0 \\
& \ddots & & \ddots & \\
0 & & \mathbf{h}(0) & \cdots & \mathbf{h}\left(L_{h}-1\right),
\end{array}\right]
$$

onde $\mathbf{h}(\lambda)=\left[h_{0}(\lambda) h_{1}(\lambda) \ldots h_{P-1}(\lambda)\right]^{T}, \quad \lambda \in$ $\left\{0,1, \ldots, L_{h}-1\right\}$, é o vetor com $\lambda$-ésima amostras de cada subcanal.

Da equação (16) podemos obter o equalizador baseado no critério zero forcing (ZF), dado por:

$$
\mathbf{W}_{Z F}=\left[\begin{array}{l}
\mathbf{I}_{P \times P} \\
-\mathbf{F}
\end{array}\right] \frac{\mathbf{h}(0)}{\|\mathbf{h}(0)\|^{2}} .
$$

Uma versão adaptativa, denominada PLP-A0, derivada do equalizador ZF (18) é proposta em [5]. O vetor de predição progressiva é estimado a cada iteração e os coeficientes da matriz de predição progressiva atualizados através do algoritmo dos mínimos quadrados (LMS), conforme apresentado na Figura 2. Tal versão foi avaliada em [6] para canais estáticos no tempo.

Uma simplificação do algoritmo PLP-A0 (Figura 2) é proposta em [7]. Tal versão elimina as etapas (3.) e (4.) ao assumir que $\widehat{\mathbf{h}}(0)=\left[\begin{array}{llll}1 & 1 & \ldots & 1\end{array}\right]^{T}$, ou seja, elimina a necessidade de estimar os primeiros coeficientes dos subcanais assumindo que os mesmos são iguais a unidade.

\section{EqualizaÇão Multiportadora por RestauraÇão DA REDUNDÂNCIA CÍCLICA - MERRY}

O algoritmo para equalização de sistemas multiportadora baseado na restauração da redundância do CP (MERRY em Inglês) assume que o CP é usado na transmissão e que a sequiência $x_{i}^{k}$, antes da inserção do $\mathrm{CP}$, é descorrelacionada [2]. O algoritmo MERRY explora a redundância inserida pelo $\mathrm{CP}$, sem necessitar de seqüência de treinamento. Desta forma, pode ser considerado autoditada ou cego.

$$
\begin{aligned}
& \text { Para } n=0,1, \ldots \\
& \text { (1.) Inicialização } n=0 \\
& \widehat{\mathbf{E}}(0)=\delta \mathbf{I}_{P \times P} \quad \text { e } \delta=0.001 \\
& \mathbf{F}(0)=\mathbf{0}_{P\left(L_{w}-1\right) \times P} \\
& \mathbf{F}(n+1)=\mathbf{F}(n)+\mu \mathbf{U}(n-1) \mathbf{e}^{H}(n) \\
& \mathbf{W}(n)=\left[\begin{array}{c}
\mathbf{I}_{P \times P} \\
-\mathbf{F}(n+1)
\end{array}\right] \frac{\hat{\mathbf{h}}(0)}{\|\hat{\mathbf{h}}(0)\|^{2}}
\end{aligned}
$$

Fig. 2. Algoritmo adaptativo para predição linear progressiva de atraso zero em canal espaço-temporal (PLP-A0).

O critério de otimização usado pelo algoritmo MERRY é baseado na minimização da diferença cíclica quadrática média, conforme descrito pela função custo dada por:

$$
\begin{gathered}
J_{M E R R Y}(\mathbf{w}, \Delta)=E\left\{\|\varepsilon(M k+\nu+\Delta)\|^{2}\right\}, \\
\varepsilon(i)=y(i)-y(i+N),
\end{gathered}
$$

com $i \in\{1,2, \ldots, \nu\}$, onde $\Delta \in\{0,1, \ldots, M-1\}$ é um parâmetro de sincronismo de símbolo.

A escolha de $\Delta$ influencia diretamente o desempenho do algoritmo. Um procedimento heurístico para determinar o valor adequado para $\Delta$ é proposto em [8].

Desconsiderando a presença de ruído, para $L_{e} \leq \nu$, onde $L_{e}$ é o comprimento do canal equivalente da convolução entre o canal e o equalizador, a última amostra do CP será igual a última amostra do mesmo símbolo. Portanto, a função custo estará sendo minimizada na medida em que os equalizadores restabelecem a redundância do $\mathrm{CP}$.

O algoritmo adaptativo MERRY é derivado tomando o gradiente da equação (19) em relação a w, condicionado à restrição de norma unitária do equalizador, de modo a evitar a solução trivial. Pelo método da descida mais íngreme, chegase ao algoritmo apresentado na Figura 3.

\section{Modelo dos Canais com Desvanecimento lento E RÁPIDO}

Na condição $\nu<L_{e}$, o desempenho dos sistemas OFDM é degradado em função da IIS. Desta forma, a degradação da informação transmitida $x(n)$ pode ser modelada como a convolução temporal

$$
y(n) \quad=x(n) \otimes h(n, \tau)+r(n),
$$

com a resposta ao impulso do canal seletivo em frequiência com desvanecimento Rayleigh modelado por:

$$
h(n, \tau)=\sum_{l=1}^{L} g_{l}(n) \delta\left(t-\tau_{l}\right),
$$


Para os símbolos $k=0,1, \ldots$

(1.) Inicialização $k=0$

$$
\mathbf{w}(0)=\left[\begin{array}{llllll}
0 & \ldots & 1 \ldots & 0 & 0 & \ldots
\end{array}\right]^{T} \quad \text { center spike }
$$

(2.) Obter o erro da redundância cíclica $\mathbf{e}(n)$

$$
\begin{aligned}
& \tilde{\mathbf{u}}_{s}(k)=\mathbf{u}_{s}(k M+v+\Delta)-\mathbf{u}_{s}(k M+v+N+\Delta) \\
& e(k)=\mathbf{w}^{T} \tilde{\mathbf{u}}_{s}(k)
\end{aligned}
$$

(3.) Atualizar coeficientes do filtro

$$
\widehat{\mathbf{w}}(k+1)=\widehat{\mathbf{w}}(k)+\mu e(k) \tilde{\mathbf{u}}_{s}^{*}(k)
$$

(4.) Normalizar o equalizador

$$
\mathbf{w}(k+1)=\frac{\widehat{\mathbf{w}}(k+1)}{\|\widehat{\mathbf{w}}(k+1)\|}
$$

(5.) Voltar ao ítem (2.)

Fig. 3. Algoritmo adaptativo para equalização de sistemas com modulação em múltiplas portadoras baseado na restauração da redundância (MERRY).

em que $L$ é o número de multipercursos, $g_{l}(n)$ é um processo aleatória complexo discreto de média nula, cuja envoltória segue uma distribuição Rayleigh, e $\tau_{l}$ é o atraso de propagação do $l$-ésimo percurso.

Paralelamente, os sistemas OFDM podem sofrer forte degradação em função da interferência inter-portadora (ICI em Inglês). Umas das possíveis causas da ICI é o desvio Doppler provocado pela mobilidade dos rádios.

Para modelar os canais com desvio Doppler, utilizamos o modelo de canal do tipo Jakes, que considera de forma explícita o desvio Doppler [1]. No modelo de Jakes, o processo aleatório associado ao $l$-ésimo mulitipercurso é a soma de exponenciais complexas com freqüências que varrem o espectro Doppler:

$$
g_{l}(n)=\sum_{k=1}^{N_{e}} e^{j\left[2 \pi f_{D} \cos \left(\phi_{k}\right) n+\Phi_{k}\right]},
$$

em que $N_{e}$ é o número de exponenciais complexas, $f_{D}$ é a freqüência Doppler máxima, $\phi_{k}$ é o ângulo de partida do sinal e $\Phi_{k}$ a fase de recepção da $k$-ésima onda. Assumimos que $\phi_{k}$ e $\Phi_{k}$ são uniformemente distribuídas no intervalo $[0,2 \pi)$.

\section{RESUltados}

Para tornar mais simples as simulações e, com isso, possibilitar uma análise mais clara das técnicas apresentas, alguns dos blocos e funcionalidades dos sistemas de comunicação foram desconsideradas. Dentre elas, podemos destacar as etapas de codificação de fonte e de canal. O sincronismo do sistema foi considerado perfeito nas simulações.

Os algoritmos foram avaliados em relação à forma como exploram a diversidade oferecida pela estrutura de múltiplas antenas, assim como o desempenho obtido em canais variantes no tempo.

\section{A. Ganho de Diversidade Espaço-Temporal em Canais Estáticos}

Um dos objetivos em se empregar múltiplas antenas tanto na transmissão quanto na recepção é explorar a diversidade espacial que tal configuração pode oferecer.

A fim de comparar os ganhos que os algoritmos MERRY e PLP-A0 possam ter em função da diversidade espacial, utilizou-se a SNR na saída do equalizador como medida do ganho de diversidade, dada por:

$$
S N R=\frac{\left\|\mathbf{h}_{e}\right\|^{2}}{\left\|\mathbf{w}_{0}\right\|^{2}+\left\|\mathbf{w}_{1}\right\|^{2}} \frac{\sigma_{x}^{2}}{\sigma_{r}^{2}},
$$

em que $\sigma_{x}^{2}$ é a potência do sinal transmitido $x(n)$ e $\sigma_{r}^{2}$ é a potência do ruído, admitindo o mesmo valor para todos os subcanais, e $\mathbf{h}_{\mathbf{e}}$ é o canal equivalente dado por:

$$
h_{e}(n)=\sum_{p=0}^{P-1} \sum_{\lambda=0}^{L_{h}-1} h_{p}(\lambda) w_{p}(\lambda-n) .
$$

A avaliação dos algoritmos se dá em dois cenários, o primeiro cenário é o caso $\nu \geq L_{h}$. No segundo cenário, os subcanais são seletivos em freqüência e os algoritmos foram avaliados para diversos valores de $\nu$. Em ambos os cenários, o número de antenas é $P=2$ e a razão $\sigma_{x}^{2} / \sigma_{v}^{2}$ fixada em $30 \mathrm{~dB}$.

Os passos de adaptação, assim como o comprimento das seqüências de treinamento, foram ajustados de tal sorte que os algoritmos atinjam a convergência para um patamar em torno de $10^{-4}$ de seus respectivos critérios de erro quadrático médio, dados por:

$$
\begin{gathered}
E Q M_{P L P-A 0}=E\left\{\|y(n)-x(n)\|^{2}\right\}, \\
E Q M_{M E R R Y}=E\left\{\|e(n)\|^{2}\right\},
\end{gathered}
$$

em que $e(n)$ é dado na Figura 3.

O parâmetro $\Delta$ (sincronismo do algoritmo MERRY) foi ajustado com o valor da posição do vetor de inicialização w $(0)$ (Figura 3) cujo valor é diferente de zero, conforme proposto em [8].

\section{- Cenário 1}

Os subcanais utilizados foram:

$$
\begin{aligned}
& \mathbf{h}_{0}=1 e^{+i \frac{\pi}{2}} \\
& \mathbf{h}_{1}=1 e^{-i \frac{\pi}{2}} .
\end{aligned}
$$

A cada simulação, um dos subcanais ou nenhum deles será multiplicado por um fator de atenuação $\alpha$ para emular as condições de diversidade de maior interesse para avaliação dos algoritmos. Cada combinação de diversidade será testada nas três possíveis inicializações em center-spike para o algoritmo MERRY. As três possíveis combinações são o primeiro, o segundo ou os dois subequalizadores inicializados com centerspike. Vale ressaltar que a inicialização de um único subequalizador com center-spike e dos demais com valores nulos foi proposta pelo próprio autor do MERRY em [9]. A inicialização com center-spike em todos os subequalizadores é uma variante proposta por nós.

Para os subcanais planos em freqüência, o desempenho do PLP-A0 é o mesmo do filtro casado (MF), conforme apresentado na Tabela I. Na condição $\nu \geq L_{h}$, o algoritmo MERRY, na média, não atualiza seus coeficientes em função de seu gradiente verdadeiro ser zero, conforme mostrado em [6]. Sendo assim, o algoritmo MERRY mantém como solução 
TABELA I

\begin{tabular}{|c|c|c|c|c|c|}
\hline Canal Atenuado & PLP-A0 & $\overline{\mathbf{M E}}$ & $\overline{\mathrm{RY} S \mathrm{SN}}$ & $(\mathrm{dB})$ & $\overline{\text { MF }}$ \\
\hline$\alpha=10^{-3}$ & $\mathrm{SNR}(\mathrm{dB})$ & {$\left[\begin{array}{ll}1 & 0\end{array}\right]$} & {$\left[\begin{array}{ll}0 & 1\end{array}\right]$} & {$\left[\begin{array}{ll}1 & 1\end{array}\right]$} & $\operatorname{SNR}(d B)$ \\
\hline$\overline{\alpha \times \mathbf{h}_{0}}$ & $\overline{30.0}$ & -13.6 & 22.1 & -12.5 & $\overline{30}$ \\
\hline$\alpha \times \mathbf{h}_{1}$ & 30.0 & 22.0 & -13.6 & -12.5 & 30 \\
\hline Nenhum & 33.0 & -9.2 & -9.4 & -10.4 & 33 \\
\hline
\end{tabular}

a inicialização empregada. Para o cenário em que um dos subcanais sofre forte atenuação, o ganho do algoritmo MERRY depende da inicialização adequada. Já para o PLP-A0, o ganho permanece em $30 \mathrm{~dB}$, independentemente do subcanal atenuado. Quando nenhum dos subcanais sofre atenuação, o ganho de SNR do PLP-A0 em relação ao algoritmo MERRY é de aproximadamente $40 \mathrm{~dB}$ para as três possíveis inicializações em center-spike do algoritmo MERRY.

\section{- Cenário 2}

Os algoritmos foram avaliados para uma série de valores do CP utilizando os seguintes subcanais:

$$
\begin{aligned}
\mathbf{h}_{0} & =\left[\begin{array}{lrrrr}
0.49 & -0.34 & 0.25 & -0.20 & 0.05
\end{array}\right]^{T}, \\
\mathbf{h}_{1} & =\left[\begin{array}{lllll}
0.49 & 0.39 & 0.29 & 0.20 & 0.15
\end{array}\right]^{T} .
\end{aligned}
$$

Conforme indicado na Tabela II, o PLP-A0 mantém praticamente o mesmo patamar de SNR, independente do tamanho do CP utilizado. Por outro lado, a SNR obtida com o algoritmo MERRY aumenta na medida em que o CP aumenta. A SNR do algoritmo MERRY, inicializado em center-spike nos dois subequalizadores, diminui consideravelmente quando comparada com as outras duas possibilidades de inicialização.

\begin{tabular}{|c|c|c|c|c|c|}
\hline $\mathbf{C P}$ & PLP-A0 & \multicolumn{3}{|c|}{ MERRY SNR(dB) } & MFB \\
\hline$\nu$ & $\mathrm{SNR}(\mathrm{dB})$ & {$\left[\begin{array}{ll}1 & 0\end{array}\right]$} & $\left.\begin{array}{ll}0 & 1\end{array}\right]$ & {$\left[\begin{array}{ll}1 & 1\end{array}\right]$} & $\mathrm{SNR}(\mathrm{dB})$ \\
\hline 2 & 26.1 & 20.4 & 20.8 & 0.7 & 31.6 \\
\hline 3 & 26.1 & 24.2 & 23.9 & 4.0 & 31.6 \\
\hline 4 & 26.1 & 24.2 & 24.0 & 8.0 & 31.6 \\
\hline 5 & 26.1 & 24.2 & 24.0 & 8.2 & 31.6 \\
\hline
\end{tabular}

TABELA II

Nos dois cenários apresentados, verificamos que o algoritmo MERRY pode atenuar significativamente o sinal desejado, caso não haja uma inicialização adequada do mesmo. Para que haja inicialização adequada, o algoritmo MERRY necessita de uma estimativa dos subcanais utilizados, o que nem sempre é possível ou desejável. Por outro lado, o PLP-A0 independe da forma como as atenuações do subcanais se apresentam. Portanto, o PLP-A0 explora melhor que o algoritmo MERRY a diversidade contida no canal.

\section{B. Desempenho dos Algoritmos em Canais Variantes no Tempo}

Em canais com desvanecimento rápido, as estimativas dos coeficientes $\mathbf{h}(0)$, obtidas pelas etapas (3.) e (4.) na Figura 2, tornam-se grosseiras, levando à perda de desempenho do PLP-A0. Em função disso, optamos por empregar o PLPA0 proposto em [7], para evitar a necessidade de estimar os coeficientes $\mathbf{h}(0)$ dos subcanais.

Para avaliar as propostas apresentadas em canais variantes no tempo, dividimos a simulação na fase de treinamento e
TABELA III

$$
\tau_{k}=k T_{s} \mathrm{E} T_{s}=1 / f_{s}=0,1 \mu s
$$

\begin{tabular}{|c|c|c|c|c|c|c|c|c|c|}
\hline \hline \multirow{2}{*}{ Grupos } & \multirow{2}{*}{$\begin{array}{l}\text { Sub-canais } \\
\text { Associados }\end{array}$} & \multicolumn{7}{|c|}{ Perfil de Potência $E\left\{\left\|g_{l}(n)\right\|^{2}\right\}$} \\
\cline { 3 - 10 } & & $\tau_{0}$ & $\tau_{1}$ & $\tau_{2}$ & $\tau_{3}$ & $\tau_{4}$ & $\tau_{5}$ & $\tau_{6}$ & $\tau_{7}$ \\
\hline \multirow{2}{*}{ I } & $\mathbf{h}_{0}$ & 0.49 & 0.34 & 0.25 & 0 & 0 & 0 & 0.20 & 0.05 \\
\cline { 2 - 10 } & $\mathbf{h}_{1}$ & 0.49 & 0.39 & 0.29 & 0 & 0 & 0 & 0.20 & 0.15 \\
\hline \hline \multirow{2}{*}{ II } & $\mathbf{h}_{0}$ & 0.22 & 0.27 & 0.38 & 0 & 0 & 0 & 0.22 & 0.05 \\
\cline { 2 - 10 } & $\mathbf{h}_{1}$ & 0.38 & 0.49 & 0.44 & 0 & 0 & 0 & 0.27 & 0.16 \\
\hline \hline
\end{tabular}

rastreamento. $\mathrm{Na}$ etapa de treinamento, o canal permaneceu estático e os algoritmos convergiram para as soluções ótimas, segundo seus respectivos critérios. Após os algoritmos atingirem a convergência, seguimos com a etapa de rastreamento do canal, em que a BER foi medida.

Nas simulações, utilizamos os perfis de potência do GrupoI e Grupo-II (Tabela III) para ponderar os desvanecimentos gerados pelo modelo de Jakes. Consideramos que os atrasos $\tau_{l}$ são múltiplos inteiros da taxa de amostragem do sistema OFDM. Os passos de adaptação empregados na etapa de treinamento foram obtidos de forma a minimizar a estimativa do erro quadrático médio para cada algoritmo. Todavia, na etapa de rastreamento, os passos de adaptação foram ajustados de tal sorte que os algoritmos possam melhor rastrear as mudanças do canal. Para o algoritmo MERRY, o passo de adaptação foi ajustado em $\mu_{M}=3.8$ e, o algoritmo PLP$\mathrm{A} 0, \mu_{P}=1.5$. O algoritmo MERRY foi inicializado com center-spike no primeiro subequalizador para os dois perfis de potência empregados.

A taxa de amostragem do simulador OFDM foi ajustada em $f_{s}=10 M H z$ e o número de portadoras empregadas foi de $N=64$. Utilizamos a modulação binary phase shift keying (BPSK) e a potência do símbolo $X_{f}^{k}$ (Figura 1) foi ajusta em $\sigma_{X}^{2}=1$, o número de coeficientes dos subequalizadores em $L_{w}=15$, desvio Doppler máximo em $f_{D}=10 \mathrm{kHz}$ e 10 milhões de bits foram transmitidos para cálculo da taxa de erro.

O desempenho dos algoritmos PLP-A0 e MERRY em canais com múltiplos percursos e variantes no tempo foi avaliado através das curvas de taxa de erro de bit (BER em Inglês) em função da SNR. Utilizamos o filtro casado (MFB em Inglês) como limitante inferior para comparação das propostas apresentadas.

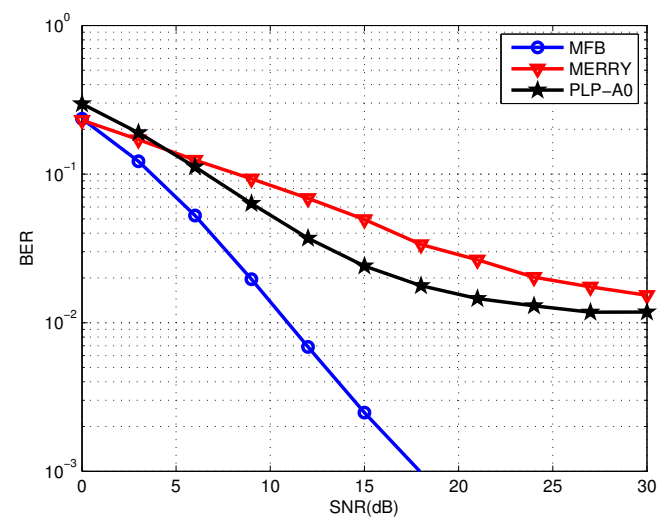

Fig. 4. G-I, $\nu=4$ e $f_{D} / f_{s}=10^{-4}$. 


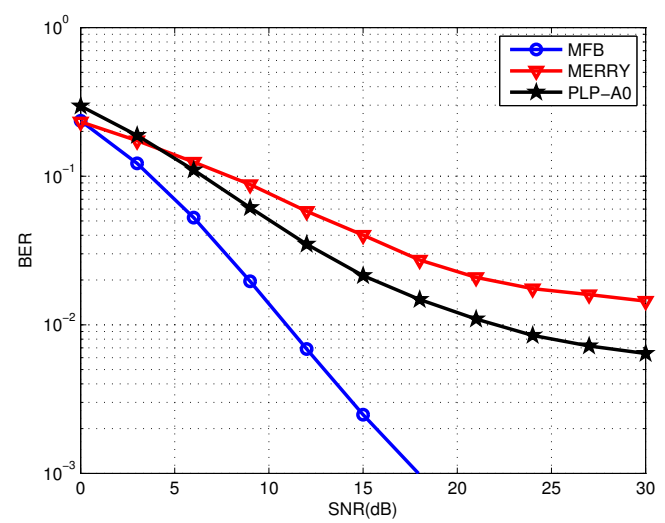

Fig. 5. G-I, $\nu=10$ e $f_{D} / f_{s}=10^{-4}$.

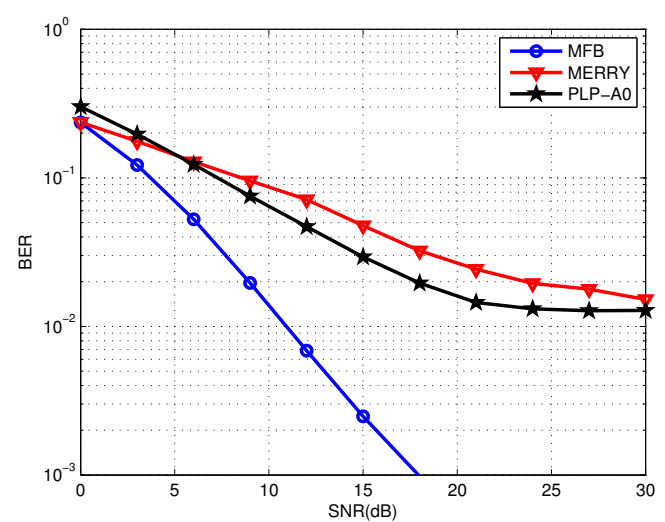

Fig. 6. G-II, $\nu=4$ e $f_{D} / f_{s}=10^{-4}$.

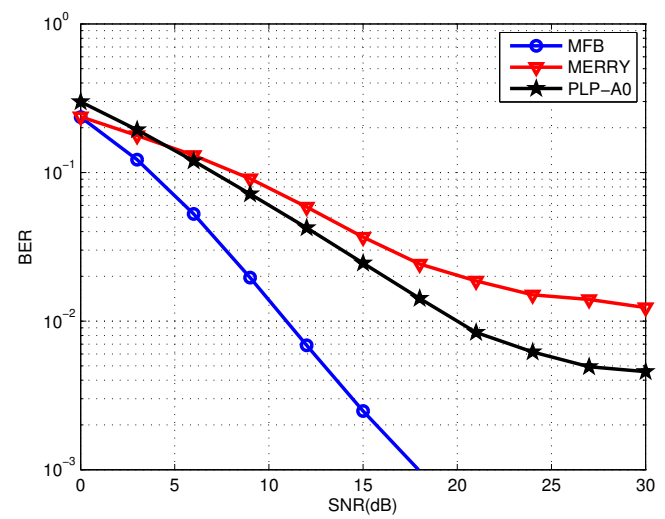

Fig. 7. G-II, $\nu=10$ e $f_{D} / f_{s}=10^{-4}$.

Na condição de desvanecimento considerada, $f_{D} / f_{s}=10^{-4}$, com $\nu \leq L_{c}$, verificamos que o ganho do PLP-A0 é aproximadamente de $9 \mathrm{~dB}$ em relação ao MERRY para uma BER de $2 \times 10^{-2}$, nas Figuras 4 e 6 . Por outro lado, na condição $\nu \geq L_{c}$, o ganho do PLP-A0 em relação ao MERRY é de aproximadamente $5 \mathrm{~dB}$ para uma BER de $2 \times 10^{-2}$, conforme verificamos nas Figuras 5 e 7.

O ganho de desempenho do PLP-A0 em relação ao MERRY está, em parte, relacionado com a velocidade de convergência dos algoritmos. A velocidade de convergência do PLP-A0 é superior ao MERRY, o que possibilita ao PLP-A0 rastrear o canal de forma mais precisa [6]. Neste caso, o PLP-A0 age como uma espécie de controle automático de ganho (CAG) mais Phase-Locked Loop (PLL), compensando a variação do canal a cada amostra. Já o algoritmo MERRY, que adapta somente a cada símbolo, não é capaz de corrigir as variações que ocorrem durante o símbolo OFDM, permitindo que ocorra ICI.

\section{CONClusões E PERSPectivas}

O presente artigo propõe a predição linear progressiva como estrutura de equalização de sistemas OFDM. Para corroborar nossa proposta, utilizamos o algoritmo MERRY como referência de comparação.

O algoritmo PLP-A0 apresenta desempenho superior ao MERRY por explorar a diversidade das múltiplas antenas. Isso se verifica para canais planos e seletivos em freqüência. Notamos também que o desempenho do algoritmo MERRY é dependente de inicialização adequada no contexto SIMO.

Em canais variantes no tempo, o ganho de desempenho do PLP-A0 em relação ao MERRY pode ser explicado em parte pela diferença na velocidade de convergência dos algoritmos. O PLP-A0 atualiza seus coeficientes a cada amostra, ao passo que o MERRY atualiza a cada símbolo OFDM.

O desempenho do PLP-A0 é degradado quando canais estáticos de fase não mínima são utilizados. Para tentar contornar esse problema, estruturas de predição linear regressiva em cascata com predição linear progressiva serão exploradas em futuros trabalhos. Além disso, ao adotarmos a simplificação proposta por [7], perdemos a possibilidade de garantir sempre o casamento de fase nas saídas dos subcanais/subequalizadores. Cremos que tal problema possa ser resolvido minimizando-se conjuntamente o erro de fase na saída dos subcanais, melhorando assim o desempenho do sistema.

\section{AgRADECIMENTOS}

Os autores gostariam de agradecer à CAPES pelo suporte financeiro.

\section{REFERÊNCIAS}

[1] W. C. Jakes, ’Microwave Mobile Communications,’Wiley-Interscience, 1974.

[2] R. K. Martin, J. Balakrishnan, W. A. Sethares, and C. R. Johnson Jr., Blind, adaptive channel shortening for multicarrier systems, in Proc. IEEE Asilomar Conf. Signals, Syst., Comput., Pacific Grove, CA, Nov. 2002.

[3] S. Haykin, ”Blind Deconvolution,"Prentice-Hall, 1994.

[4] C. B. Papadias and D. T. M. Slock, Fractionally Spaced Equalization of Linear Polyphase Channels and Related Blind Techniques Based on Linear Prediction, IEEE Transactions on Signal Processing, vol. 47, No. 3, pp. 641-654, March 1999.

[5] X. Li, H. Fan, Linear prediction methods for blind fractionally spaced equalization, IEEE Trans. Signal Processing, vol. 48, no. 6, pp. 1667 1675, Junho 2000.

[6] A. S. Menezes, C. M. Panazio, J. M. T. Romano, Blind Channel Shortening for SIMO Space-Time Channels Using Linear Prediction in OFDM Systems, International Telecommunications Symposium 2006, Fortaleza, Brasil.

[7] M. S. Castro, J. M. T. Romano, Adaptive Approaches for Blind Equalization Based on Multichannel Linear Prediction, International Telecommunications Symposium 2002, Natal, Brasil.

[8] R. K. Martin, J. M. Walsh, C. R. Johnson Jr., Low complexity MIMO blind, adaptive channel shortening, IEEE Trans. Signal Processing, vol. 53, no. 4, pp. 1324 - 1334, Apr. 2005.

[9] R. K. Martin, Joint Blind Adaptive Carrier Frequency Offset Estimation and Channel Shortening, IEEE Trans. Signal Processing, vol. 54, no. 11, pp. 4194-4203, Nov. 2006. 